\title{
Coherent backscattering of turbid samples containing large Mie spheres
}

\author{
Ralf Lenke $^{1,2}$, Ralf Tweer ${ }^{1}$ and Georg Maret ${ }^{1}$ \\ ${ }^{1}$ University of Konstanz, Box 5560, D-78457 Konstanz, Germany \\ ${ }^{2}$ Institut Charles Sadron, 6 rue Boussingault, F-67083 Strasbourg Cedex, France
}

\begin{abstract}
We found that the coherent backscattering cone of turbid samples containing spherical Mie scatterers with a size parameter larger than about 20 strongly deviates from known analytical curve shapes. We compare experimental data with numerical simulations of Monte Carlo type. Moreover, we present a new wide-angle coherent backscattering set-up.
\end{abstract}

Keywords: Multiple scattering of light, coherent backscattering, Mie scattering

\section{Introduction}

Coherent backscattering (CB) is the constructive interference in the exact backscattering direction between each light path in a multiple scattering sample and its reversed path [1-6]. The angular width of the so-called CB cone is proportional to the wavelength $\lambda_{0}$ in air and inversely proportional to the transport mean free path $\ell^{\star}$, which is the characteristic length of a photon random walk in a turbid medium. $1 / \ell^{\star}$ is a measure for the turbidity of the sample. Out of the exact backscattering direction, i.e. with increasing 'backscattering' vector $^{3} q_{b}$, the twofold intensity enhancement of CB decreases, on average over all light paths, to the 'incoherent background', which is usually normalized to unity. In a scalar diffusion approximation the cone shape, i.e. the coherent backscattering enhancement $E_{C B}\left(\vec{q}_{b}\right)$, of a non-absorbing sample as a function of $\vec{q}_{b}$ is given by [2]

$$
E_{C B}\left(\vec{q}_{b}\right) \simeq 1+\begin{gathered}
1-\exp \left[-2(1+\gamma)\left|\vec{q}_{b}\right| \ell^{\star}\right] \\
2(1+\gamma)\left|\vec{q}_{b}\right| \ell^{\star}
\end{gathered} .
$$

The first term represents the incoherent background, the second term the angular-dependent interference between direct and reversed paths. Here, we have neglected single scattering, which does not contribute to $\mathrm{CB}$ and reduces the maximum

\footnotetext{
3 In analogy to the forward-scattering angle $\theta=/\left(\vec{k}_{\text {in }}, \vec{k}_{\text {out }}\right)$, we define the 'backscattering' angle $\theta_{b}=/\left(-\vec{k}_{\text {in }}, \vec{k}_{\text {out }}\right)$ as the angle between the reversed incident wavevector $-\vec{k}_{\text {in }}$ and the outgoing wavevector $\vec{k}_{\text {out }}$ with $|\vec{k}|=2 \pi / \lambda_{0}$ (elastic scattering). The backscattering vector $\vec{q}_{b}$ is given by the difference $\vec{k}_{\text {out }}-\left(-\vec{k}_{\text {in }}\right)$ with $\left|\vec{q}_{b}\right| \stackrel{\theta_{b}}{\approx} \approx 00 \theta_{b} / \lambda_{0}$
}

enhancement factor of two. Approximation (1) is only valid if incident and detected polarization states are the same because only those channels fulfil the theorem of reciprocity [7]. In the orthogonal channels no or only a small CB cone is observed. In this paper, we shall only consider the case of the same incident and detected circular polarized light ' ++ ', which is completely symmetric in the azimuthal angle of $\vec{q}_{b}$ as implicitly suggested by equation (1). The factor $\gamma \approx 0.7$ in equation (1) is known from radiative transport theory in the context of the 'Milne problem' [8]. Using a random walk model as we do, it simply reflects the fact that a multiple-scattering light path with a given average step length $\ell^{\star}$ does not end exactly on the interface of the sample but at a certain distance of order $\ell^{\star}$ outside the sample. The average value of this distance is just $\gamma \ell^{\star}$ [3]. In the Monte Carlo simulations $\gamma$ does not appear.

Comparative CB measurements of samples with varying $\ell^{\star}$ are easily possible. Quantitative evaluation, however, is not straightforward as diffusion approximations such as equation (1) are only a first-order approach for the backscattering geometry. Experimental details must also be taken into consideration, such as the polarization of the incident and detected light [9], internal reflections at the surface of the sample $[10,11]$, the type of scattering [12] or the sample geometry. Normally, we find a better coincidence with experiments by comparison with Monte Carlo simulations [3].

In this paper we shall study, experimentally as well as by Monte Carlo simulations, the $\mathrm{CB}$ cone of samples consisting of large Mie spheres, a case which holds true for many emulsions, such as mayonnaise [13], for example. We shall realize that the CB cone of these samples consists to about $20 \%$ of a 


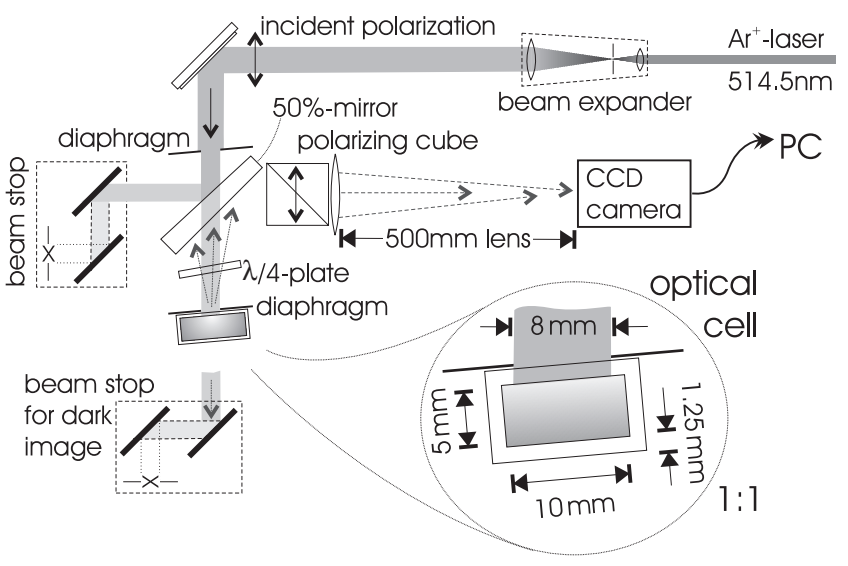

Figure 1. Small-angle CB set-up (see text).

part which decreases very slowly with increasing $q_{b}$, strongly deviating from equation (1). This behaviour may easily result in a misinterpretation of the experimental data. However, the experimental findings are well reproducible by Monte Carlo simulations, from which we shall learn that a relatively large amount of double scattering accounts for this unexpected behaviour.

We shall first present the experimental set-ups and the experimental results. Then we discuss the experimental findings and the role of double scattering. In section 5 we explain the simulations in more detail and discuss a numerical problem related to scattering by large Mie spheres.

\section{Experimental set-ups}

We have used two different experimental set-ups: a standard 'small-angle' set-up for an angle range of $0^{\circ}$ to about $\pm 1.5^{\circ}$ and a 'wide-angle' set-up for angles larger than $0.05^{\circ}$ (see figures 1 and 2, respectively). The standard set-up is described in earlier works [3] in more detail. It includes an enlarged parallel, linear polarized laser beam (total $1 / e$ beam width $=$ $8 \mathrm{~mm}$ ), a semi-transparent mirror and a CCD camera. A $\lambda / 4-$ phase retarding plate allows circular polarized incidence and detection. The samples consisted of an optical cell containing water suspensions of colloidal polystyrene spheres [14] with diameters up to $10 \mu \mathrm{m}$. They were strongly agitated just before each measurement in order to avoid sedimentation. During the measurement, the optical cell was slightly wiggled by hand directly behind the diaphragm to improve the averaging out of the speckles. The second diaphragm is necessary to guarantee that each detected light path is propagated through in both directions. The CCD camera had a linear response and created images of $512 \times 512 \times 1$ bytes at video rate. We averaged over 255 images. A dark (averaged) image, created by removing the sample and using the second beam stop, was subtracted from each measurement in order to obtain absolute intensity values. The final data curves were obtained by averaging the images in the azimuthal angle around the cone maximum.

The wide-angle set-up makes use of the fact that CB (and the Glory, see below) act in some sense like a phase conjugating mirror. When using divergent incident light from a pointlike (image) source, CB is produced around it with its maximum at the very position of the light source [3]. In the set-up, we use a circular polarized laser beam which is focused at

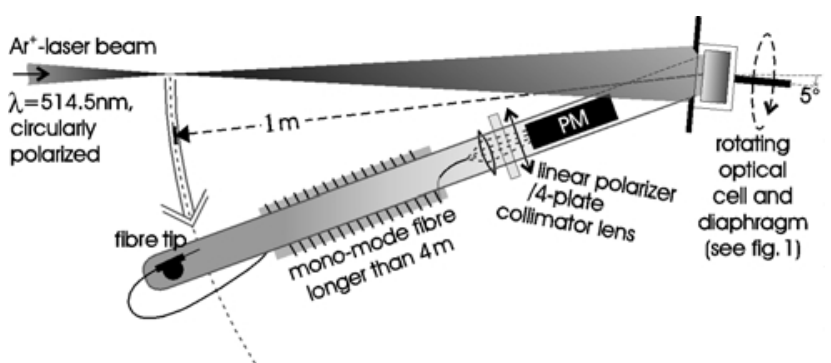

Figure 2. Wide-angle CB set-up (see text). The optical elements and the photomultiplier (PM) are mounted at the bottom of the goniometer arm. The sample is twisted by $5^{\circ}$ with respect to the incident light in order to avoid direct reflection.

a point $1 \mathrm{~m}$ in front of the sample. A mono-mode fibre, at least $4 \mathrm{~m}$ long, some optics and a photomultiplier are rigidly mounted on a goniometer arm, whose centre lies underneath the sample. The bare fibre tip travels on a circle through the focal point of the laser beam. The mono-mode fibre preserves the circular polarization, which is analysed via a $\lambda / 4$-plate and a linear polarizer just before the photomultiplier. During the measurement, which took about $1 \mathrm{~h}$, the sample was rotated at about $1 \mathrm{~Hz}$ to avoid sedimentation. Note that if we define the focal point as the light source and the fibre tip as the detection point, no optical elements are placed between light source, sample and detector in this set-up. CB measurements with this wide-angle set-up of samples with very small $\ell^{\star}$, i.e. with a very broad cone, are reported in [15].

\section{Experimental details}

Figure 3 shows a CB-measurement series of suspensions of polystyrene spheres with three different size parameters $k a$ in water. The maxima of the cones do not reach the theoretical value of two, for which there are several reasons: first, due to the incident Gaussian laser beam profile, the amplitudes of the direct and reversed paths can be different. Our simulations result, for the given geometry and $\ell^{\star}$-values, in a maximum enhancement factor of about 1.98. Another reason is single scattering, which, however, is suppressed in the case of circular polarization and spherical scatterers and therefore does not reduce the enhancement factor here. Obviously, the main reason for a reduced cone height is the limited resolution of our experimental set-up (see section 2), camera and camera optics. As the experimentally obtained exact enhancement factor is not known, we usually normalize the measured curves by the intensity at large angles, i.e. at angles of about ten times the cone width out of exact backscattering. With our small-angle set-up, we normally obtain enhancement factors of about 1.8 in the case of $\ell^{\star}$-values of the order of $300 \mu \mathrm{m}$ [3]. This is also the value obtained here, for the sample with the smallest size parameter $(k a=1.71)$. For this sample, the measured cone shape is quite similar to that given by equation (1) after instrumental correction, i.e. convolution with the system response. However, the measured cone width is reduced by about $15 \%$ with respect to equation (1), which may be explained by the influence of the internal reflections (see section 1).

For the larger spheres, normalization with respect to the incoherent background is not possible: in the case of $k a=33.1$ 


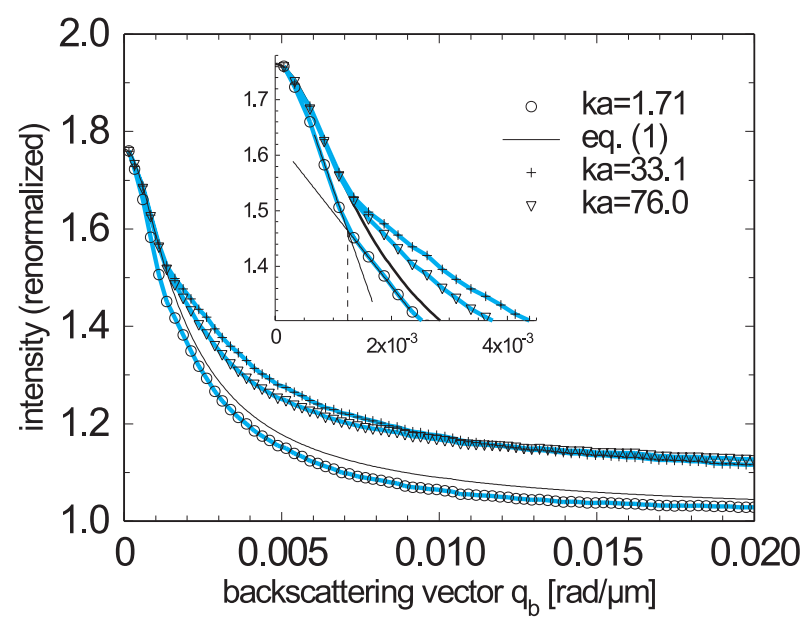

Figure 3. Measured backscattering intensity as a function of $q_{b}$ for three suspensions of polystyrene spheres with different size parameters in water (circular polarization, $\lambda_{0}=0.5145 \mu \mathrm{m}$ ). The curves are averaged in the azimuthal part of $\vec{q}_{b}$. Circles: sphere radius $a=0.105 \pm 0.005 \mu \mathrm{m}$, volume fraction $\phi=0.0061$, $\ell_{\text {theo }}^{\star}=330 \mu \mathrm{m}$ calculated from Mie theory. Crosses: $a=2.03 \pm 0.035 \mu \mathrm{m}, \phi=0.041, \ell_{\text {theo }}^{\star}=245 \mu \mathrm{m}$. Triangles: $a=4.66 \pm 0.04 \mu \mathrm{m}, \phi=0.096, \ell_{\text {theo }}^{\star}=314 \mu \mathrm{m}$. Solid black curve: equation (1) for $\ell^{\star}=330 \mu \mathrm{m}$, convoluted with $\exp \left[-\left(q_{b} / 5.9 \times 10^{-4}\right)^{2}\right]$ as an approximation for system response. The solid grey curves are guidelines to the eye. Inset: enlargement close to exact backscattering. The change in the slope at $q_{b} \approx 1.25 \times 10^{-3}$ is caused by reflections inside the glass window of the optical cell. Note that the curves for $k a=33$ and 76 are rescaled such that the enhancement factor at $q_{b}=0$ is equal to that of the smallest scatterers (see the text).

no flat background is detectable within the measuring range $\left(q_{b}<0.1 \mathrm{rad} \mu \mathrm{m}^{-1}\right)$ and for $k a=76.0$ normalization with respect to the supposed background would result in an unexpectedly small enhancement factor of 1.59 . Therefore, in order to compare the cone shapes of the three samples, we rescaled the curves of the larger spheres to the same value at $q_{b}=0$ as for the sample with the smallest scatterers. As explained above, this procedure is justified by the fact that all three samples had about the same $\ell^{\star}$-value, thus nearly the same slope of the cone for small $q_{b}$, and consequently they should have, after convolution with the instrumental system response, nearly the same maximum value at $q_{b}=0$. This cone shape is also confirmed by Monte Carlo simulations (for more details see section 5) as can be seen in figure 4, where best coincidence is found for an enhancement factor of 1.85 . Moreover, the cone shape suggested in figure 4 is in good agreement with the wide-angle measurements as can be seen in figure 5 .

At backscattering angles $\theta_{b}>2^{\circ}$ (figure 5) the oscillating single-particle form factor $\mathrm{d} \sigma$ superimposed on the incoherent multiply scattered background becomes visible. In the backward direction, $\mathrm{d} \sigma$ essentially follows the square of a second-order Bessel function [16] $J_{2}^{2}\left(k_{0} \theta_{b} a^{\prime}\right)$ with $a^{\prime}$ fitted to $1.38 a$ in this case. (For more details on the value of $a^{\prime}$ as well as the relationship between the $\mathrm{CB}$ cone and this typical form factor, also known as the (backward) Glory, see [6].) Note that in the exact backscattering direction, in the case '++', single scattering by spherical particles is suppressed.

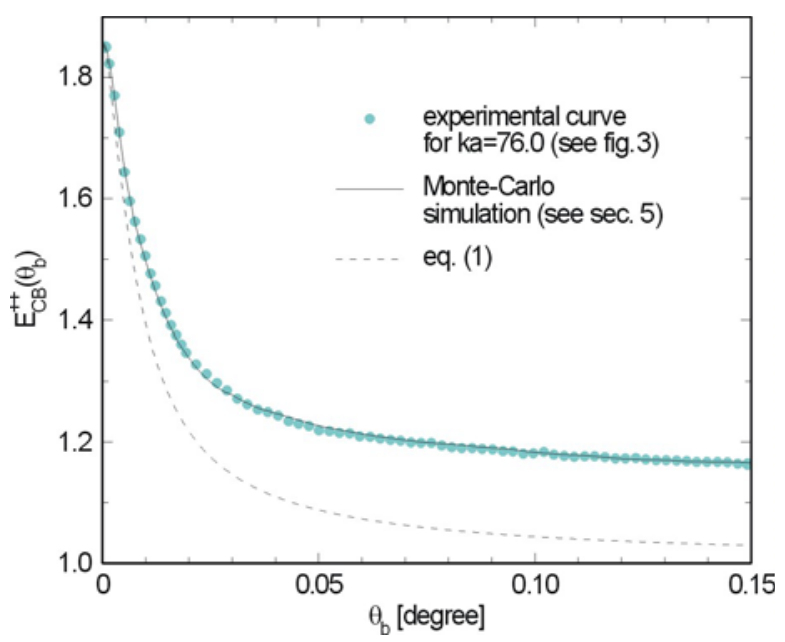

Figure 4. $\mathrm{CB}$ enhancement as a function of backscattering angle $\theta_{b}$. Points: the same experimental curve as in figure 3 for $k a=76.0$ but rescaled according to the simulations. Dashed curve: numerical simulation (for more details see section 5). The measured intensity was normalized such that it matches the simulated curve at $\theta_{b}=0.15^{\circ}$, which corresponds to $q_{b} \ell^{\star}=10 \mathrm{rad}$. The simulated curve was convoluted with a Gaussian, $\exp \left[-\left(q_{b} / 5.1 \times 10^{-4}\right)^{2}\right]$, such that the enhancement factors at $\theta_{b}=0$ are equal. This corresponds to about the same system response as in figure 3 . No other fit parameter was used. The simulations took into account the experimental boundary conditions and used the $\ell^{\star}$-value of $314 \mu \mathrm{m}$ obtained by Mie theory.

\section{Discussion of experimental results}

The results of the evaluation procedure in the previous section suggest that the $\mathrm{CB}$ cone of large Mie spheres as a function of $q_{b}$ is composed of a 'normal' fast decreasing part and a slowly decreasing part originating from an increased probability of light paths with starting and end points lying very close together. This cone shape was also confirmed by Monte Carlo simulations (figure 4). Therefore, the question of the origin of these narrow loops arises. Figure 6 shows the scattering profile of the large polystyrene spheres ${ }^{4}$. According to figure 6(b) about $45 \%$ of the light is scattered very close to the forward direction into an angle range $\theta \leqslant 2^{\circ}$. This part of the light does not contribute much to the randomization of the photon random walk and therefore could also be attributed to the coherent, nonscattered beam (see section 5). In fact, when taking this weakly scattered part out from the differential scattering cross section $\mathrm{d} \sigma$, which can easily be done in the simulations, the slowly decreasing wings of the cone disappear and the cone shape essentially resembles that given by equation (1). However, this cannot be the only reason as Mie spheres with a ratio of the inner to outer index of refraction $m=n_{i} / n_{0}<1$ (inverse structure) do not show these raised wings [5]. The second reason is the relatively large amount of light being scattered in and around the exact backscattering direction (the Glory), which is only very pronounced in the case $m>1[6,16]$. Both effects together result in an increased probability of double-scattering events where the light is mainly scattered in the forward direction during the first scattering event and in the backward direction $\left(-\vec{k}_{i n}\right)$, out of the sample, during the second scattering event. Start and end points of these

4 The calculations have been performed using the 'Mie Scattering User Interface', Valley Scientific Inc. (1998). 


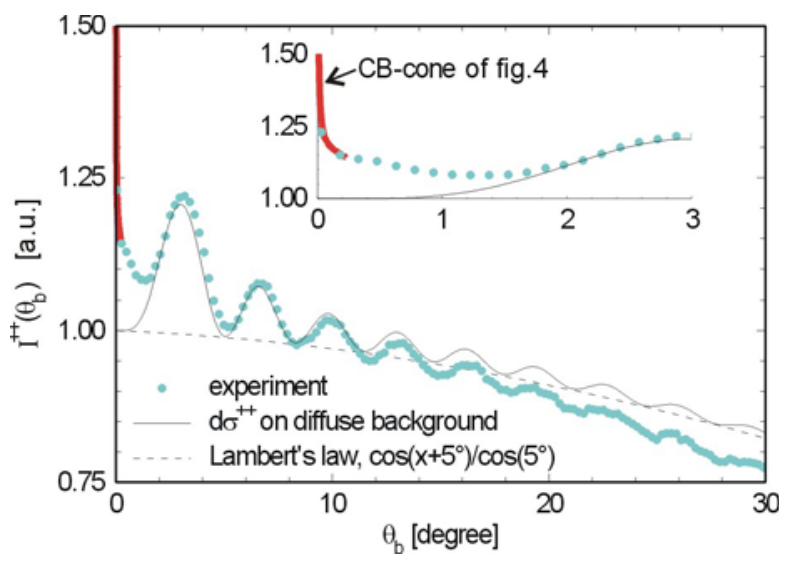

Figure 5. Backscattered intensity $I^{++}$at larger angles where the scattering profile of single spheres is visible. Points: experimental data measured with wide-angle set-up (see section 3), rescaled such that the intensity coincides with the wings of the $\mathrm{CB}$ cone at smaller angles (red curve). Dashed curve: Lambert's law. Note that the sample was twisted by $5^{\circ}$ with respect to $\vec{k}_{i n}$. Dotted curve: $\mathrm{d} \sigma^{++}$ from Mie theory (see figure 6(a)) on diffuse background, corrected by Lambert's law. The curve was rescaled by

$\theta_{b} \rightarrow \arcsin \left[n_{\text {water }} \sin \left(\theta_{b}+3.74^{\circ}\right)\right]-5^{\circ}$ in order to account for the refraction at the plane entrance window. The fitted ratio of the diffuse normalized background to the differential cross section normalized by the total cross section was $1: 0.52$. In the calculations we did not account for shadowing effects of the diaphragm and the angular dependence of the internal reflections. Therefore, the experimental curve decreases faster at larger angles $\theta_{b}$ than the calculations.

light paths lie very close together, resulting in a wide-angle contribution to the $\mathrm{CB}$ cone. Numerically, this explanation was verified by excluding the double-scattering events from the cone profile in the simulations, with the result that the raised wings of the cone disappeared.

\section{Simulations}

In the Monte Carlo simulations we use a random walk model combined with the technique of 'partial photons', which is very suitable for studying the phenomenon of $\mathrm{CB}$. We took care of the dimensions of the optical cell $\left(n_{\text {glass }}=1.526\right)$ and the laser beam profile (see figure 1). Internal reflections at and in the glass plates of the optical cell have also been taken into consideration in the simulations using Fresnel's laws. We have also tested the influence of the (small) polydispersity of our samples (see the appendix), which, however, did not show any significant effect here.

The implementation of these simulations has already been described in more detail elsewhere $[3,4]$. Here, we want to focus on a numerical problem, which is related to the narrow forward-scattering peak in $\mathrm{d} \sigma$ of large Mie scatterers (see figure 6). In the case of Mie scatterers with a size parameter $k a \leqslant 5$ or in the case of Rayleigh-Gans-Debye scattering, it is sufficient to average over $10^{5}$ photons, normally. For $k a \geqslant 20$, however, the simulated curves are not very smooth even after $10^{6}$ photons. This problem disappears if the strongly forward-scattering peak is cut out of $\mathrm{d} \sigma$ and treated as light which was not scattered: for example, in the case of figure 6 , scattering angles $\theta<2^{\circ}$ are attributed to the non-scattered coherent beam. This modification only slightly changes the
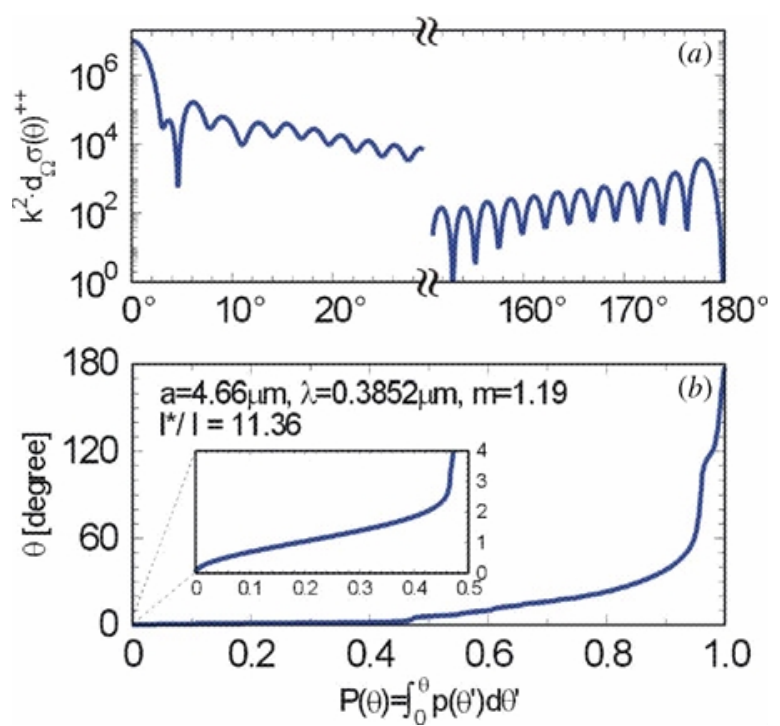

Figure 6. (a) Differential cross section for the given polystyrene spheres and circular polarized light. (b) In $[3,4]$ we have defined a function $p(\theta)=\max \left(\left|\mathrm{S}_{1}\right|^{2},\left|\mathrm{~S}_{2}\right|^{2}\right)$, where $\mathrm{S}_{1 / 2}$ are the amplitudes of the Mie scattering matrix. The integral of this curve is used to choose the scattering angles during the simulation according to their weight. For small angles the absolute values of both coefficients are very similar. Consequently, this figure essentially shows the integrated probability of the light being scattered into an angle range smaller than $\theta$. Both figures demonstrate the existence of a strongly forward-scattering peak and the backward Glory. Note that in the case of $m<1$ the Glory part is orders of magnitude smaller.

characteristics of the random walk and essentially results in the same reflected intensity distribution $I(\vec{R})$, where $\vec{R}$ is the distance vector between start and end point of a light path. In fact, this trick has successfully been used before [5], in the case of Mie scatterers with $m<1$. It reduces the number of photons in the simulations again to $10^{5}$.

However, as already mentioned in section 3, this trick is only successful for $m<1$, whereas for $m>1$ the slowly decreasing wings of the cone disappear as well. As explained further above, the high wings are caused by doublescattering events with $|\vec{R}| \ll \ell^{\star}$. Using the modified cross section, these double-scattering events are replaced by singlescattering events, which do not contribute to CB. In order to also circumvent this numerical problem for $m>1$, one could try to use the modified scattering cross section only after the second scattering event, but here we used another approach.

Actually, the large noise in the simulations is strongly related to the technique of partial photons. At each scattering event of the random walk, we calculate the intensity of the light being scattered in the exact backscattering direction multiplied by the exponentially decreasing probability of reaching the surface of the sample. These 'partial photons' are added up to obtain ${ }^{6} I(\vec{R})$. The cone shape is obtained

5 Moreover, the simulation time is further reduced because the non-isotropy of the modified differential cross section, i.e. the ratio between $\ell^{\star}$ and the scattering mean free path $\ell$, is smaller. The calculation time scales with $\ell^{\star} / \ell$ because the number of scattering events scales with $\ell$ whereas the cone width essentially scales with $\ell^{\star}$. In our case, the non-isotropy of 11.4 for the unmodified cross section is reduced by a factor of about two.

6 This procedure can also be interpreted in the following way: the Monte Carlo simulations reproduce the intensity and energy flux distribution inside the sample, from which the flux outside the sample in the direction $-\vec{k}_{\text {in }}$ is calculated. 


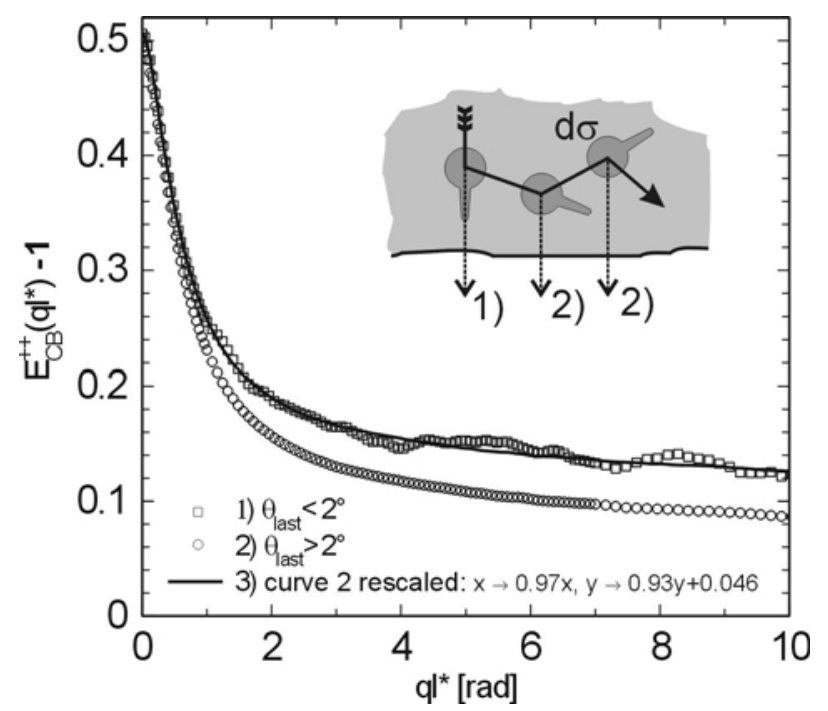

Figure 7. Monte Carlo simulation of a $\mathrm{CB}$ cone in the case of large Mie spheres ( $a=4.66 \mu \mathrm{m}, k a=76.0, m=1.19$, absorption length $\ell_{a}=100 \ell^{\star}$, circular polarization). The output of the simulations is divided into two parts representing fractions of the cone obtained by partial photons which did (curve 1) or did not (curve 2) originate from the forward-scattering peak of d $\sigma$, i.e. $\theta_{\text {last }} \lessgtr 2^{\circ}$. Both possibilities are illustrated in the sketch where the dashed lines are the partial photons. The sum of curve 2 and rescaled curve 2 (=curve 3) was compared with the experimental data in figure 4. A 'normal', straightforward simulation would result in the sum of curves 1 and 2 with a larger statistical error. Note that for clarity we did not include experimental details (sample thickness etc) here.

by Fourier transformation. From time to time the partial photons originate from light that was scattered in the narrow forward peak of $\mathrm{d} \sigma$ (see the sketch in figure 7). These events are about two orders of magnitude less frequent than partial photons, which did not belong to the forward-scattering peak. However, they contribute to a very high, non-negligible intensity (see figure 6(a)) and therefore deteriorate the statistics considerably. In figure 7 we have separately plotted the fractions of the cone originating from partial photons which have been scattered in an angle range $\theta_{\text {last }} \leqslant 2^{\circ}$ and $\theta_{\text {last }}>2^{\circ}$ during the last scattering event. The latter gives a smooth curve after $10^{5}$ photons which, as we found empirically, can be fitted by rescaling in the coordinates to the curve with the larger statistical error obtained from partial photons with $\theta_{\text {last }} \leqslant 2^{\circ}$. Finally, the sum of curves 2 and 3 can be compared with the experimental data in a satisfying way. Using this trick we essentially obtain the same statistical error as in simulations for smaller scatterers. Otherwise, the calculations would last about two orders of magnitude longer.

\section{Summary}

We have shown, experimentally as well as by numerical simulations, that the $\mathrm{CB}$ cone of Mie spheres with a large size parameter strongly deviates from that of other multiplescattering samples due to the interplay between a strongly forward-scattering peak and the Glory part in the differential cross section. The Glory itself, i.e. the single-particle scattering cross section, was measured using a new wide-angle set-up. In our cases, the transport mean free path $\ell^{\star}$ was much larger than the particle radius $a$. Therefore, the CB cone and the Glory could be studied separately. Note that this is not true generally.

The simulations are in excellent agreement with the experimental data. This was only possible by taking the dimensions of the sample into consideration, as $\ell^{\star}$ was relatively large. Internal reflections at and in the window of the optical cell have also been taken into account.

In order to calculate the Fresnel coefficients at the surfaces of the sample we took the index of refraction of water $\left(n_{\text {water }}=\right.$ 1.336) and not the average index of the emulsion $(n=1.36)$. This is justified by the facts that the scatterers we used are relatively large and that there is a certain repulsion between the glass windows of the optical cell and the colloids. For the rescaling of the Glory part in figure 5 we also used $n_{\text {water }}$. Due to the noise in our measurements at angles between $20^{\circ}$ and $30^{\circ}$ the difference of $n_{\text {water }}$ versus $n$ could not be studied here. However, reducing the noise in the measurements should not be too complicated. This could be an interesting possibility to study light propagation in a turbid medium close to a surface, which is still a problem which is not well understood, especially at higher volume fractions or larger contrasts of the indices of refraction.

\section{Acknowledgment}

This work was supported by the Optikzentrum Konstanz.

\section{Appendix. Simulation of polydisperse suspensions}

We have seen above that the cone shape strongly depends on the size parameter. A real sample is never completely monodisperse. At least, the particles have a certain particle size distribution but the sample may also contain particles of different size ranges. The simulations necessitate tables for the differential cross section of the scatterers. Consequently, for reasons of calculation time and memory capacity the number of scatterers which can be used in one simulation at the same time is limited. Fortunately, each particle size distribution can be replaced by two auxiliary scatterers. There is even some freedom in the choice of the elements of the two scattering matrices and we can set one matrix equal to identity multiplied by a real number. If $\left\{\left\{\mathrm{S}_{1}(a), 0\right\},\left\{0, \mathrm{~S}_{2}(a)\right\}\right\}$ are the scattering matrices for the particles with radius $a$ in the sample, the two matrices $S=\left\{\left\{S_{1}, 0\right\},\left\{0, S_{2}\right\}\right\}$ and $U=\{\{u, 0\},\{0, u\}\}$ representing the auxiliary scatterers must fulfil the following relations, according to the possible products in the expressions for the intensity:

$$
\begin{gathered}
\int\left|\mathrm{S}_{1}(a)\right|^{2} p(a) \mathrm{d} a=\left(\left|\mathrm{S}_{1}\right|^{2}+u^{2}\right) / 2 \\
\int\left|\mathrm{S}_{2}(a)\right|^{2} p(a) \mathrm{d} a=\left(\left|\mathrm{S}_{2}\right|^{2}+u^{2}\right) / 2 \\
\int \mathrm{S}_{1}(a) \mathrm{S}_{2}^{*}(a) p(a) \mathrm{d} a=\left(\mathrm{S}_{1} \mathrm{~S}_{2}^{*}+u^{2}\right) / 2
\end{gathered}
$$

where $p(a)$ is the normalized size distribution in the given sample and $\left(^{*}\right)$ the complex conjugate. Note that there is still some freedom in the phase angle. These relations are exact but we found that another set of equations, which corresponds to circular eigenstates, gives numerically more stable results: 


$$
\begin{gathered}
2 \int\left|\mathrm{S}_{1}+\mathrm{S}_{2}\right|^{2} p(a) \mathrm{d} a=\left|\mathrm{S}_{1}+\mathrm{S}_{2}\right|^{2}+(2 u)^{2} \\
2 \int\left|\mathrm{S}_{1}-\mathrm{S}_{2}\right|^{2} p(a) \mathrm{d} a=\left|\mathrm{S}_{1}-\mathrm{S}_{2}\right|^{2} \\
2 \int\left(\mathrm{S}_{1}+\mathrm{S}_{2}\right)\left(\mathrm{S}_{1}^{*}-\mathrm{S}_{2}^{*}\right) p(a) \mathrm{d} a=\left(\mathrm{S}_{1}+\mathrm{S}_{2}\right)\left(\mathrm{S}_{1}^{*}-\mathrm{S}_{2}^{*}\right) .
\end{gathered}
$$

The rate at which $\mathrm{S}(\theta)$ or $\mathrm{U}(\theta)$ are chosen during the simulations is given by the ratio of their total scattering cross section $\sigma_{\text {tot }}$.

\section{References}

[1] van Albada M P and Lagendijk A 1985 Phys. Rev. Lett. 55 2692-5

Wolf P-E and Maret G 1985 Phys. Rev. Lett. 55 2696-9

[2] Akkermans E, Wolf P-E and Maynard R 1986 Phys. Rev. Lett. 56 1471-4

[3] Lenke R and Maret G 2000 Multiple scattering of light: coherent backscattering and transmission Scattering in Polymeric and Colloidal Systems ed W Brown and K Mortensen (London: Gordon and Breach) pp 1-73

[4] Lenke R and Maret G 2000 J. Eur. Phys. B 17 171-85

[5] Lenke R, Lehner R and Maret G 2000 Europhys. Lett. 52 $620-6$

[6] Lenke R, Mack U and Maret G 2002 J. Opt. A: Pure Appl. Opt. at press

[7] Saxon D S 1955 Phys. Rev. 100 1771-5

[8] Morse P M and Feshbach H 1953 Methods of Theoretical Physics (New York: McGraw-Hill)

[9] Mishchenko M I 1992 J. Opt. Soc. Am. A $9978-82$

[10] Lagendijk A, Vreeker R and de Vries P 1989 Phys. Lett. A 136 81-7

[11] Saulnier P M and Watson G H 1992 Opt. Lett. 17 946-8

[12] Amic E, Luck J M and Nieuwenhuizen Th M 1996 J. Phys. A: Math. Gen. 29 4915-55

[13] Bijnen F G C, van Aalst H, Baillif P Y, Blonk J C G, Kersten D, Kleinherenbrink F, Lenke R and vander Stappen M L M 2002 Powder Technol. at press

[14] Micro particles GmbH Research and Development Laboratory, Volmesstr. 9A/ UTX, 3-51 D-12489, Berlin, Germany

[15] Bechinger C, Bertsch M and von Grünberg H H (eds) 2000 Soft Condensed Matter annual report (University of Konstanz)

[16] van de Hulst H C 1957 Light Scattering by Small Particles (New York: Dover) 